SLAC-PUB-6076 NOVEMBER 1992

(SSRL-M)

\section{CHARACTERIZATION OF THE B/SI SURFACE ELECTRONIC STRUCTURES}

R. Cao, X. Yang, and P. Pianetta

Stanford Linear Accelerator Center, Stanford Synchrotron Radiation Laboratory Stanford Synchrotron Radiation Laboratory
Stanford University, Stanford, CA 94309

\section{Abstract}

High resolution angle resolved core level and valence band photoelectron spectroscopy have been used to characterize the electronic structures of the $\mathrm{B} / \mathrm{Si}(111)-(\sqrt{3} \times \sqrt{3})$ surfaces. The results have been compared with theoretic calculations and other group III metals and $\mathrm{Si}$ terminated $\mathrm{Si}(111)$ surfaces that share the same type of surface reconstruction. We have observed a structure evolution from $B-\mathrm{T}_{4}$ to $\mathrm{B}-\mathrm{S}_{5}$ and finally to $\mathrm{Si}-\mathrm{T}_{4}$ as deposited boron atoms diffuse into the substrate with increasing annealing temperature. The chemically shifted component appearing in the Si $2 p$ core level spectrum is attributed to charge transfer from the top layer $\mathrm{Si}$ and $\mathrm{Si}$ adatoms to the sublayer B-S 5 atoms. For the $\mathrm{Si} / \mathrm{Si}(111)-(\sqrt{3} \times \sqrt{3})$ surface, a newly discovered chemically shifted component is associated with back bond formation between the $\mathrm{Si}$ adatoms and the underneath $\mathrm{Si}$ atoms. A new emission feature has been observed in the valence band spectra unique to the $\mathrm{B} / \mathrm{Si}(111)-(\sqrt{3} \times \sqrt{3})$ surface with $\mathrm{B}-\mathrm{S}_{5}$ configuration. Thin Ge layer growth on this structure has also been performed, and we found that no epitaxial growth could be achieved and the underneath structure was little disturbed.

Work supported by the U.S. Deparument of Energy under Contract DE-AC03-76SF00515

Presented at the 39th AVS National Symposium, Chicago, IL, November 9-13, 1992

\section{Introduction}

$B / S i$ surfaces have received much attention recently. For boron on $\mathrm{Si}(111)$ surface, although as other Group-III metal atoms (e.g. Al, $\mathrm{Ga}$, and In), it can form stabilized ( $\sqrt{3} \times$ $\sqrt{ } 3) R 30^{\circ}$ reconstruction, where these metal atoms are located on the $T_{4}$ adatom sites, that is, the adatoms are located on the top of the second layer Si atoms, it distinguishes itself by the capability of forming the same type of reconstruction but with an unique configuration. [1-4] A number of studies using scanning tunneling microscopy (STM), $x$-ray diffraction as well as low energy energy diffraction have recently determined that the thermally stablest configuration for $\mathrm{B}$ on $\mathrm{Si}(111)$ is that boron atoms occupy $\mathrm{S}_{5}$ sites (i.e. $\mathrm{Si}$ is this case occupy the $T_{4}$ sites while boron atoms are located right underneath the $S i$ adatoms). [4-7] This configuration has also been predicted by theoretical calculations. [8] Such a reconstruction can be generated by annealing the B-covered surface and/or Si substrate with high $B$ doping concentration at high temperature. It is of great interest from scientific point of view to understand the electronic structure of this new surface structure, where photoemission can play a major role.

Another interesting aspect of the $\mathrm{B} / \mathrm{Si}$ structure is that it provides the opportunity to create an atomically sharp doping layer, so-called delta doping, by growing epitaxial Si overlayer on top of this structure. $[9,10]$ In fact, it has been reported recently that such a delta doping structure can be realized for the $\mathrm{B} / \mathrm{Si}(100)$ when the $\mathrm{B}$ layer underneath the epitaxially grown $\mathrm{Si}$ film is $100 \%$ electrically activated. [9] It offers a great opportunity in semiconductor industrial applications. The B/Si structure also offers possibility of forming the atomic scale negative differential resistance device. [11] Therefore, not only does such a structure bear scientific interest, it has great potential in industrial applications as well.

\section{MASTER}

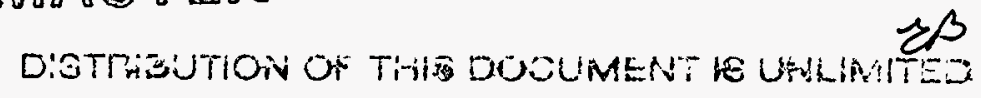


In this work, we carried out a high resolution photoelectron spectroscopic study of the $B / S i$ structure focusing on understanding of the electronic structure of the $B / S i(111)-(\sqrt{3} \times \sqrt{3})$ surface, particularly the modification of the electronic structure as the deposited metal atoms going from $T_{4}$ configuration to $S_{5}$ configuration. The results are also compared with other group III metal atoms as well as Si terminated $\operatorname{Si}(111)-(\sqrt{3} \times \sqrt{3})$ surfaces, where these atoms occupy only the $T_{4}$ sites. $[1-3,12,13]$ Growth of Ge overlayer on the B/Si structure was also conducted and compared with the growth on other group-III metal terminated surface. It is one of few model systems to investigate the growth condition and the role of surface energy plays during the epitaxial growth process. A similar study has also been carried out for the $\mathrm{B} / \mathrm{Si}(100)-(2 \times 1)$ structure although we will concentrate on the results and discussing of the $B / S i(111)-(\sqrt{ } 3 \times \sqrt{3})$ systems in this paper. Some of the results from the $\mathrm{B} / \mathrm{Si}(100)$ studies will be mentioned when appropriate while the detailed studies will be published elsewhere.

\section{Experimental}

The experiments were conducted at the Stanford Synchrotron Radiation Laboratory (SSRL) on both beam line III-1 with a grasshopper monochromator and beam line VIII-1 with a toroidal grating monochromator. A VG ADES 400 angle resolved photoemission ultra vacuum chamber equipped with a hemispherical spectrometor and an angle integrated photoemission system with a double pass cylindrical analyzer (CMA) were used. The combined energy resolution was less than $0.25 \mathrm{eV}$ for the angle resolved system with angle resolution about $2^{\circ}$, where Si $2 p$ core level and valence band spectra were taken. The surface reconstruction was characterized by an in situ LEED optics. The Si(111) wafers were cleaned before inserted into the UHV systems with base pressure around $2 \times 10^{-10}$ torr. They were outgassed at $\sim 600^{\circ} \mathrm{C}$ and at low pressure for few hours. The clean

-3. surfaces were finally generated by beating up to $1000^{\circ} \mathrm{C}$ for short period of time. Sharp (7 $\times 7)$ LEED patterns were observed afterwards. The high temperature annealing was achieved through an e-beam heating. Boron deposition was accomplished through evaporation of solid $\mathrm{B}_{2} \mathrm{O}_{3}$ mounted on a tungsten filament while the $\mathrm{Si}$ wafers were held at about $500^{\circ} \mathrm{C}$ during deposition, and the thickness was calibrated by the evaporation rate read from an in situ crystal thickness monitor. The photoemission spectra and LEED show that a $700^{\circ} \mathrm{C}$ post annealing desorbs oxygen from the system and generates a sharp $(\sqrt{3} \times$ $\sqrt{3}$ )R300 surface reconstruction. This reconstruction sustains with the annealing temperature up to $950^{\circ} \mathrm{C}$. We will show later on that although the LEED pattern does not change in this temperature range the detailed atomic arrangement can change rather drastically. After $700^{\circ} \mathrm{C}$ annealing, a $\mathrm{B} / \mathrm{Si}(111)-(\sqrt{3} \times \sqrt{3})$ structure is obtained with part of the $B$ atoms still occupying the top surface $T_{4}$ sites. As the temperature increases to around $800^{\circ} \mathrm{C}$, majority of the $\mathrm{B}$ atoms diffuse into the sublayer and occupy the substitutional $S_{5}$ sites. Finally, if one raises the temperature even higher (above $900^{\circ} \mathrm{C}$ ), all the $B$ atoms diffuse further into the bulk, leaving the $(\sqrt{3} \times \sqrt{3})$ reconstruction stabilized only by $\mathrm{Si}$ atoms. All the annealing mentioned previously lasted approximately about 10 $\min$ in these experiments. No contamination has been detected through the entire process.

\section{Results and Discussions}

\section{(A) Core level spectroscopy}

Showing in figure 1 are the $B$ is core level spectra ( $h v=220 \mathrm{eV}$ ) of $B$ on the $S i(111)$ surface as a function of annealing temperature. The top spectrum is the $\mathrm{B}$ 1s when $\mathrm{B}_{2} \mathrm{O}_{3}$ was deposited on the $\mathrm{Si}(111)$ surface beld at $500^{\circ} \mathrm{C}$. In this case, $\mathrm{B}$ atoms are mainly bound to oxygen atoms. After $700^{\circ} \mathrm{C}$ annealing, a rather large shift of the $\mathrm{B}$ 1s peak about .4 . 


\section{DISCLAIMER}

Portions of this document may be illegible in electronic image products. Images are produced from the best available original document. 
$5 \mathrm{eV}$ towards the lower binding energy is observed. At the same time, the photoemission spectra indicate that the substrate surface is free of oxygen. Meanwhile, sharp $(\sqrt{3} \times$ $\sqrt{3})$ R30 $0^{\circ}$ LEED pattern is observed. These results essentially indicate that the $B-O$ bonds are broken while oxygen desorbs from the surface and all the $B$ atoms are incorporated with the substrate Si atoms, consistent with a recent report by Weir et al. [14] Oxygen desorption at a rather low temperature $\left(<700^{\circ} \mathrm{C}\right)$ has also been reported previously when $\mathrm{HBO}_{2}$ was used as the source for the B deposition. [10] The large shift of the B 1s core level position results from the fact that B-O bond is mainly ionic with charge transferred from $B$ atom to $O$ atom, while the B-Si bond is covalent. As the annealing temperature increases, the $B$ 1s remains at roughly the same position but the intensity starts to decrease. The reduction of intensity is most likely due to $B$ diffusion into the substrate. When the temperature is raised over $900^{\circ} \mathrm{C}$, very little amount of $\mathrm{B}$ can been detected, indicating that most $B$ has diffused into the bulk. We would like to remind the readers that the $(\sqrt{3} \times \sqrt{3})$ LEED pattern remains unchanged through a wide temperature range between $700^{\circ} \mathrm{C}$ and $950^{\circ} \mathrm{C}$, particularly at the high temperature end, no B can be detected from the substrate with photoemission. If one raises the temperature even higher (over $1000^{\circ} \mathrm{C}$ ) the original $(\sqrt{3} \times \sqrt{3})$ pattern is replaced by a $(1 \times 1)$ LEED pattern, and from spectroscopic point of view the new surface looks in many aspects similar to the clean Si surface.

Now let us take a look at the Si $2 p$ core level spectra shown in figure 2. All the spectra have been decomposed using a computer least square fitting. The fitting parameters are approximately the same as those we have used before. [15] Spectrum (A) is typical of the clean $\mathrm{Si}(111)-(7 \times 7)$ surface. Beside the bulk component sitting in the middle, there are two surface components on the two sides and it is generally established that they originate from the surface Si atoms. After B deposition followed by $700^{\circ} \mathrm{C}$ annealing, two surface states seen in curve (A) vanish. Now the computer fitting shows that the new spectrum consists of two components $0.45 \mathrm{eV}$ apart. When we varied the photoelectron probing depth by changing the incident photon energies, the low binding energy component is identified as the contribution from the substrate, while the high binding energy one is assigned as a B-related Si component. As stated before, this time the surface already shows a $(\sqrt{3} \times \sqrt{3})$ reconstruction and all the deposited boron atoms are incorporate with the substrate. When the annealing temperature is increased from $700^{\circ} \mathrm{C}$ to $800^{\circ} \mathrm{C}$, LEED pattern remains unchanged, and one sees increase of intensity of the B-related component. It is also interesting to note that the B-related component is even stronger in intensity than that of the bulk component. Here we further argue that this component originates from the Si which bonds to $B$ atoms that sit on the $S_{5}$ sites. STM study suggests that with low temperature annealing surface boron atoms can occupy $\mathrm{T}_{4}$ sites, as other group-III metal atoms. [4] It is essentially because formation of the B-S 5 configuration involves break of the substrate Si-Si bonds. Therefore even B-S 5 is the most thermodynamically stable structure predicted by the theoretical calculations it is not a kinetically favorable one. While the boron atoms occupy the $\mathrm{T}_{4}$ sites, they basically saturate the three Si dangling bonds without creating a new one, a behavior shared by all other group-III metals. Spectroscopically, the original surface states of the clean Si surface vanish and no additional component will appear. This is exemplified by spectrum $(E)$ that is taken from a Ga-terminated Si- $(\sqrt{3} \times \sqrt{3})$ surface. On the contrary, when B occupies the subsurface $S_{5}$ site, a hole is generated. This prompts a charge transfer from $\mathrm{Si}$ which is located at $\mathrm{T}_{4}$ site to the hole at the B-S 5 site. Such a charge transfer is reflected by the new chemically shifted component shown in the Si $2 p$ core level spectra in figure 2. When the temperature is raised from $700^{\circ} \mathrm{C}$ to $800^{\circ} \mathrm{C}, \mathrm{B}$ 1s intensity decreases, while intensity of the B-related Si component increases. This is in accordance with $B$ diffusion from surface to the substrate and increased population of the B-S $\mathrm{S}_{5}$ sites. Our finding is consistent with the work by McLean et al. [16] In their work the $B / S i(111)-(\sqrt{3} \times \sqrt{3})$ surface was prepared by 
annealing a heavily B-doped Si samples. More annealing cycles would place more boron atoms into the $\mathrm{S}_{5}$ sites and as the result, and stronger B-related $\mathrm{Si}$ component was seen.

Another interesting observation is the intensity ratio between the B-related Si component to the bulk Si component. The results of the computer fitting show that this ratio is larger than one. Similar behavior has been also observed by McLean et al in their photoemission work. [16] The simple probing depth analysis indicates that not only the Si adatoms which occupy the $T_{4}$ sites now transfer charges to the boron atoms which occupy the $S_{5}$ sites, the $\mathrm{Si}$ layer between boron atoms and $\mathrm{Si}-\mathrm{T}_{4}$ atoms are also likely to be involved. In fact, recent Si cluster calculation suggests that the charge redistribution occurs in the top layer as a result of back bond formation, and this could lead to a situation that $\mathrm{Si}$ adatom and its near Si neighbor are in a more or less the equivalent state. [17] If one assumes that all the four $\mathrm{Si}$ neighbors in the top layers (three in the surface layer plus one adatom) donate the same amount of charge to the boron atom, the total transferred charge determined from our measurement turns out to be about $4 \times 1 / 4=1$ electron. [18]

Further increase of the annealing temperature up to $950^{\circ} \mathrm{C}$ results in depletion of $\mathrm{B}$ in the top few layers of the $\mathrm{Si}$ wafer as illustrated in figure 1 although LEED pattern remains the same, and we believe that now the surface reconstruction is stabilized by Si atoms only. Changes in the Si 2 p spectrum are obvious. The B-bonded Si component is replaced by the surface component resulting from the $\mathrm{Si} / \mathrm{Si}(111)-(\sqrt{3} \times \sqrt{3})$ surface. The separation between this new component and the bulk component position decreases a little. This is consistent with fact that less charge transfer may be involved in this type of configuration. Also, the intensity of this component is considerably lower compared with the B-S configuration. Finally the observed broadening of the component suggests that there exists certain degree of the inhomogeneity on this surface and possibly some unresolved components.

\section{(B) Angle resolved valence band spectroscopy}

Figure 3 presents the angle resolved valence band spectra $(\mathrm{hv}=17 \mathrm{eV})$ of the $\mathrm{B} / \mathrm{Si}(111)$ $(\sqrt{3} \times \sqrt{3})$ surface with $B$ occupies the $S_{5}$ site and the spectra were recorded along both $\bar{\Gamma}$.

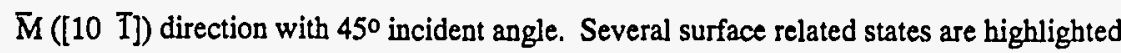
by the tic-marks. The dispersion of these surface related features shows periodicity of the surface $(\sqrt{3} \times \sqrt{3})$ Brillouin zone and is consistent with the recently published work by Grehk et al. [19] They also show the similar trend as those in other group-III/Si(111)-( $\sqrt{3}$ $x \sqrt{3})$ surfaces. The key difference between the $B / S i(111)-(\sqrt{3} \times \sqrt{3})$ surface and the other group-III/Si $(111)-(\sqrt{3} \times \sqrt{3})$ surfaces is the observation of the characteristic surface state $A_{4}$ for the $B / S i(111)-(\sqrt{ } 3 \times \sqrt{3})$ but not for other surfaces.

It is interesting to compare the surface electronic structure of the $(\sqrt{3} \times \sqrt{3})$ surface but stabilized by different atoms. Shown in figure 4 are the valence band spectra at normal emission for $\mathrm{Ga} / \mathrm{Si}(111)-\mathrm{T}_{4}, \mathrm{~B} / \mathrm{Si}(111)-\mathrm{S}_{5}$, and $\mathrm{Si} / \mathrm{Si}(111)-\mathrm{T}_{4}$. For sake of comparison, the spectrum for the $\mathrm{Si}(111)-(7 \times 7)$ is also included. Notice that the $\mathrm{A}_{3}$ peak located at the same position for all the surfaces listed in this figure except $\mathrm{Ga} / \mathrm{Si}-(\sqrt{3} \times \sqrt{3})$. It is worth pointing out that for those surfaces $\mathrm{Si}$ adatoms are located at $\mathrm{T}_{4}$ sites and $\mathrm{A}_{3}$ feature has back bond nature. The surface state $A_{4}$, which is about a few tenth of $\mathrm{eV}$ in the higher binding energy direction to the surface state $A_{3}$, is only observed on the $B / S i-(\sqrt{3} \times \sqrt{3})$ surface and we believe that is associated with the B-S 5 site. This observation appears to be consistent with the result of the theoretical calculation that total energy of the B-S configuration is lower (about $1 \mathrm{eV}$ ) than that of the $T_{4}$ structure. [8] The surface related .8 . 
feature $A_{1}$ can be seen from the $B / \operatorname{Si}(111)-(\sqrt{3} \times \sqrt{3})$ surface but its intensity varies for different surface treatment. In general, lower temperature annealing gives rise to unnoticed amount of contribution while this feature becomes noticeable at high temperature. Such a feature has also been observed for some of the group-III/Si(111) $-(\sqrt{3} \times \sqrt{3})$ surfaces. [2] Theoretical calculations show that the $\Sigma_{1}$ band (corresponding to the $A_{1}$ state in the valence band spectra) is empty for all the group-III/Si(111)- $(\sqrt{3} \times \sqrt{3})$ surfaces while it can be partially filled for $\mathrm{Si} / \mathrm{Si}(111)-(\sqrt{3} \times \sqrt{3})$ surface. $[12,13]$ STM studies have revealed that the group-III/Si(111) $-(\sqrt{3} \times \sqrt{3})$ surfaces are usually not ideal and the $\operatorname{Si} / \operatorname{Si}(111)-(\sqrt{3} \times \sqrt{3})$ is a common defect site on these surfaces. $[4,20]$ Therefore, we conclude that the observed $A_{1}$ feature is associated with the $\operatorname{Si} / \operatorname{Si}(111)-(\sqrt{3} \times \sqrt{3})$ sites. Large increase in intensity of the $A_{1}$ feature in the valence band spectrum of the Si stabilized $(\sqrt{3} \times \sqrt{3})$ structure certainly confirms out assignment and is consistent with the theoretical calculations.

\section{(C) Overlayer Growth}

We also tried to grow $\mathrm{Ge}$ on the $\mathrm{B} / \mathrm{Si}(111)-(\sqrt{3} \times \sqrt{3})$ structures. It is, however, found that no ordered Ge layer could be grown on this structure and the Ge overlayer is in an amorphous state while deposited and after low temperature annealing $\left(<500^{\circ} \mathrm{C}\right)$. Higher temperature annealing of this structure results in $\mathrm{Ge}$ island formation. It is interesting to point out that the substrate $(\sqrt{3} \times \sqrt{3})$ reconstruction is still preserved, suggesting that such a reconstruction is highly thermally stable. In contrast, when $\mathrm{Ge}$ is deposited on the $\mathrm{Ga}$ terminated $(\sqrt{3} \times \sqrt{3})$ surfaces and after mild annealing, the substrate structure is largely disrupted. Most $\mathrm{Ga}$ segregates from the interface while the deposited $\mathrm{Ge}$ atoms diffuses in to make direct contact with the substrate $\mathrm{Si}$ and to form $(5 \times 5)$ reconstruction, which has been observed for direct Ge deposition on the clean $\operatorname{Si}(111)-(7 \times 7)$ surface. The difference of these two growth modes roots from the surface energy of the substrate surfaces The B/Si(111)- $(\sqrt{3} \times \sqrt{3})$ structure has much lower surface energy compared with clean $\mathrm{Si}$ and Ge so that one would expect formation of cluster and islands when Si or Ge is deposited on this structure. On the other hand, the Ga terminated surface appears to have higher surface energy compared with either Si or Ge. More uniform layer is expect to grow on this structure. With the capability that Ga tends to diffuse out, leaving the remaining dangling bonds to be filled by the incoming species, it has the potential to act like surfactant in the Si, Ge epitaxial growth. In fact, it has been reported recently that one might be able to epitaxially grow Si and Ge layer on a slightly modified Ga-terminated Si surface. [21]

Although it is unlikely that one could grow epitaxially $\mathrm{Si}$ and Ge film on the B/Si(111)-( $\sqrt{3}$ $\times \sqrt{3}$ ) surface to accomplish delta doping, uniform amorphous $\mathrm{Si}$ and $\mathrm{SiO}_{\mathrm{x}}$ layer has been recently grown on this structure, and it is interesting to notice that the buried $B$ layer does turn into acceptor so that the delta doping can be partially accomplished in this case. [10] It should be pointed out that delta doping could be achieved on other B-related Si surface, that is the $\mathrm{B} / \mathrm{Si}(100)-(2 \times 1)$ surface. Headrick $e$ al has reported that epitaxial Si layer could be grown on this structure and the B layer turns to acceptor almost 100\%. [9] We have grown Ge epitaxial layers on this structure with the help of Sb surfactant and achieved high degree of epitaxy. It appears that this might be right direction to follow in future fabrication of the delta doping devices.

\section{Conclusions}

We have studied $\mathrm{B} / \mathrm{Si}(111)-(\sqrt{3} \times \sqrt{3})$ structures generated by depositing $\mathrm{B}_{2} \mathrm{O}_{3}$ followed by high temperature annealing. As a function of annealing temperature, the system goes from B terminated structure (B occupies $T_{4}$ sites) to sublayer $B$ stabilized structure (B occupies .10 . 
$S_{5}$ sites while Si occupies $T_{4}$ sites) and finally to pure $S i$ stabilized structure. The electronic structures have been studied and we have found that when $B$ occupies $T_{4}$ sites all the Si surface dangling bonds are saturated while there is no resolvable new chemically shifted $\mathrm{Si}$ component in the core level spectra. For B-S 5 configuration, a large chemically shifted Si component has been observed, and it is concluded that in this case both Si adatoms and the surface layer $\mathrm{Si}$ are involved as a result of charge redistribution. Finally, for the Si stabilized $(\sqrt{3} \times \sqrt{3})$ reconstructing, a new chemically shifted component is seen, which originated from the Si adatoms. The valence band structures for all the $(\sqrt{3} \times \sqrt{3})$ reconstructions are found to be similar to each other in terms of the main feature as well as their dispersion as a function of surface momentum. A distinct feature from $\mathrm{B}-\mathrm{S}_{5}$ structure $\left(A_{4}\right)$ has been seen, which has not observed for other $(\sqrt{3} \times \sqrt{3})$ structures, and we tentatively assign it to be $S_{5}$ site related features. Finally, we have grown $\mathrm{Ge}$ layers on the B-S $S_{5}$ structure and compared it with the growth on the Ga-terminated structure. It is found that no epi-growth could be achieved for the B-S $S_{5}$ structure and this structure is highly thermally stable. On the other hand, epitaxial growth can be accomplished on the $\mathrm{B} / \mathrm{Si}(100)-(2 \times 1)$ structure with the buried $\mathrm{B}$ layer acting like acceptor so that delta doping could be achieved.

\section{Acknowledgment}

The work was done at SSRL that is operated by the Department of Energy, Division of Chemical Sciences. The authors also acknowledge the support of the office's Division of Material Science for this research. One of the authors (RC) thanks J.E. Northrup for belpful discussion.

\section{References}

1. R.I.G. Uhrberg, G.V. Hansson, J.M. Nicholls, P.E.S. Persson, and S.A. Flodström, Phys. Rev. B 31, 3805 (1985).

2. T. Kinoshita, S. Kono, and T. Sagawa, Solid State Commun. 56, 681 (1985).

3. J.M. Nicholls, P. Mårtensson, G.V. Hansson, and J.E. Northrup, Phys. Rev. B 32, 1333 (1986).

4. I.W. Lyo, E. Kaxiras, and Ph. Avouris, Phys. Rev. Lett. 63, 1261 (1989).

5. P. Bedrossian, R.D. Meade, K. Mortensen, D.M. Chen, J.A. Golovchenko, and D. Vanderbilt, Phys. Rev. Lett. 63, 1257 (1989).

6. R.L. Headrick, I.K. Robinson, E. Vlieg, and L.C. Feldman, Phys. Rev. Lett. 63, 1253 (1989).

7. H. Huang, S.Y. Tong, J. Quinn, and F. Jona, Phys. Rev. B 41, 3276 (1990).

8. E. Kaxiras, K.C. Pandey, F.J. Himpsel, and R.M. Tromp, Phys. Rev. B 41, 1262 (1990).

9. R.L. Headrick, B.E. Weir, A.F.J. Levi, B. Freer, J. Bevk, and L.C. Feldman, J. Vac. Sci. Technol. A 9, 2269 (1991).

10. T. Tatsumi, I. Hirosawa, T. Niino, H. Hirayama, and J. Mizuki, Appl. Phys. Lett. 57, 73 (1990).

11. I.-W Lyo and Ph. Avouris, Science 245, 1369 (1989).

12. J.E. Northrup, Phys. Rev. Lett. 53, 683 (1984).

13. J.E. Northrup, Phys. Rev. Lett. 57, 154 (1986).

14. B.E. Weir, R.L. Headrick, Q. Shen, L.C. Feldman, M.S. Hybertsen, M. Needels, M. Schlüter, and T.R. Hart, presented on the PCSI 19, Death Valley, CA 1992.

15. R. Cao, X. Yang, J. Terry, and P. Pianetta, Phys. Rev. B 45,13749 (1992).

16. A.B. McLean, L.J. Terminello, and F.J. Himpsel, Phys. Rev. B 41, 7694 (1990).

17. W. Daum, H. Ibach, and J.E. Müller, Phys. Rev. Lett. 59, 1593 (1987). 
18. Here we assume that the $1.8 \mathrm{eV}$ shift in the Si $2 \mathrm{p}$ core level corresponds to one electron charge transfer from the Si atom after F.J. Himpsel, B.S. Meyerson, F.R. McFeely, J.F. Morar, A. Taleb-Ibrahimi, and J.A. Yarmoff, Proceedings of the International School of Physics "Enrico Fermi", edited by M. Campagna and R. Rossi (North Holland, Amsterdam, 1990), Course CVII, P. 203.

19. T.M. Grehk, P. Mårtensson, and J.M. Nicholls, Phys. Rev. B 46, 2357 (1992).

20. R.J. Hamers and J.E. Demuth, Phys. Rev. Lett. 60, 2527 (1988).

21. M. Copel, M. Horn-von Hoegen, F.K. LeGoues, and R.M. Tromp, presented on the 38th AVS Symposium, Seattle, WA 1991.

\section{DISCLAIMER}

This report was prepared as an account of work sponsored by an agency of the United States Government. Neither the United States Government nor any agency thereof, nor any of their employees, makes any warranty, express or implied, or assumes any legal liability or responsibility for the accuracy, completeness, or usefulness of any information, apparatus, product, or process disclosed, or represents that its use would not infringe privately owned rights. Reference herein to any specific commercial product, process, or service by trade name, trademark, manufacturer, or otherwise does not necessarily constitute or imply its endorsement, recommendation, or favoring by the United States Government or any agency thereof. The views and opinions of authors expressed herein do not necessarily state or reflect those of the United States Government or any agency thereof.

\section{Figure Captions}

Figure 1. The B 1 s core level spectra taken at $h v=220 \mathrm{eV}$ as a function of annealing temperatures.

Figure 2. The Si $2 \mathrm{p}$ core level spectra, taken at $\mathrm{hv}=150 \mathrm{eV}$, of (A) the clean Si(111)$(7 \times 7)$ surface, $(B)$ the $B / S i(111)-(\sqrt{3} \times \sqrt{3})$ surface annealed at $7000 \mathrm{C}$, while some $B$ still occupies the $T_{4}$ site; $(C)$ the $B / S i(111)-(\sqrt{3} \times \sqrt{3})$ surface annealed at $800^{\circ} \mathrm{C}$, while $B$ occupies the $S_{5}$ site; and $(D)$ the $S i / s i(111)-(\sqrt{3} \times \sqrt{3})$ surface annealed at $950^{\circ} \mathrm{C}$. The $\mathrm{Si}$ $2 p$ core level of the $\mathrm{Ga} / \mathrm{Si}(111)-(\sqrt{3} \times \sqrt{3})$ surface is also presented $(\mathrm{E})$.

Figure 3. The angle revolved valence band spectra as a function of photoelectron emission angle (incident angle $45^{\circ}$ ), taken at $h v=17 \mathrm{eV}$, of the $\mathrm{B} / \mathrm{Si}(111)-(\sqrt{3} \times \sqrt{3}$ ) surface with B occupying the $S_{5}$ site.

Figure 4. The valence band spectra taken at $17 \mathrm{eV}$ photon energy and normal emission (hv $=17 \mathrm{eV}$, incident angle $\left.45^{\circ}\right)$, of (A) Si(111)-(7 $\times 7$ ), (B) B/Si(111) $-(\sqrt{3} \times \sqrt{3}),(\mathrm{C})$ $\mathrm{Si} / \mathrm{Si}(111)-(\sqrt{3} \times \sqrt{3})$, and (D) $\mathrm{Ga} / \mathrm{Si}(111)-(\sqrt{3} \times \sqrt{3})$ surfaces. 


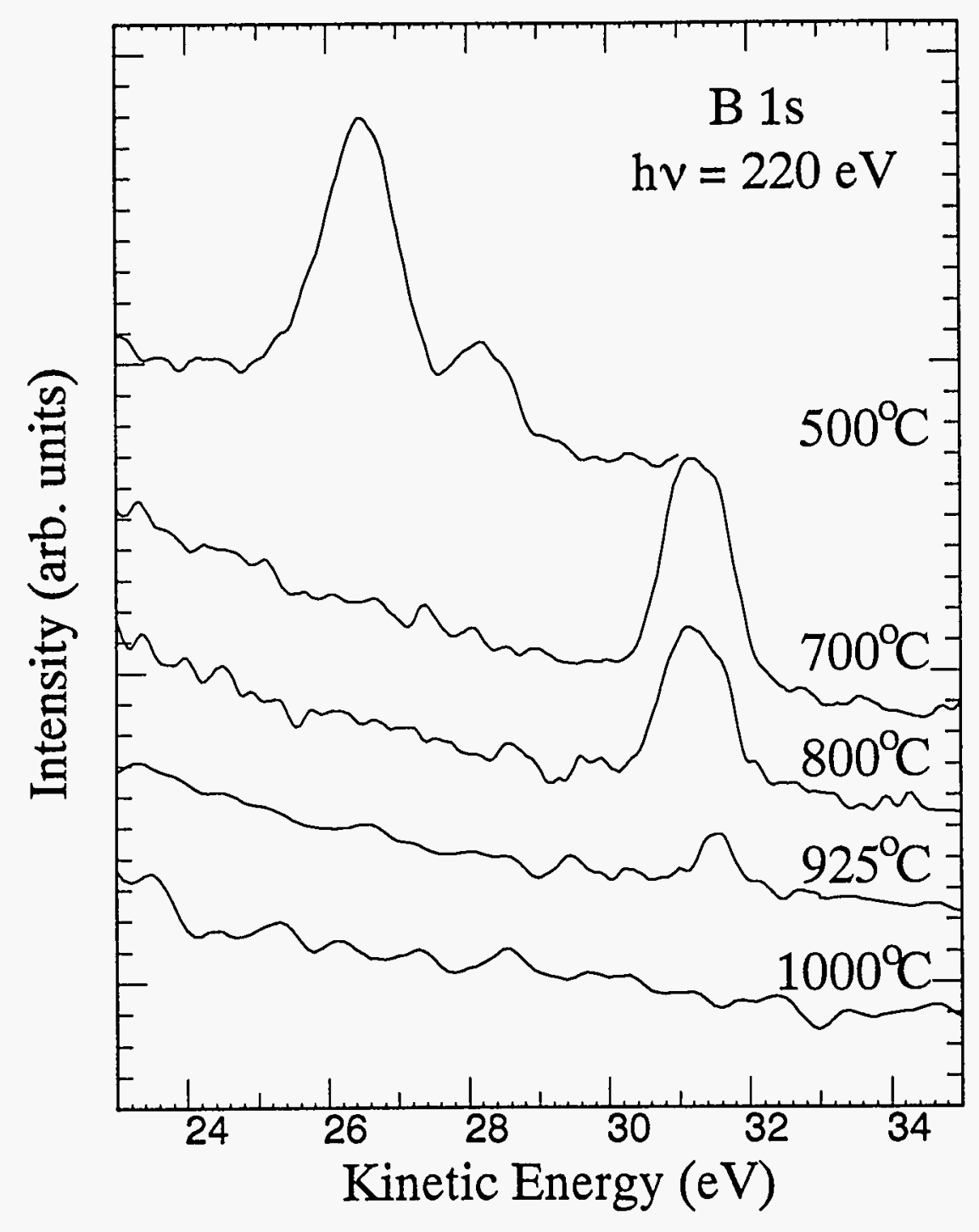

Fig. 1

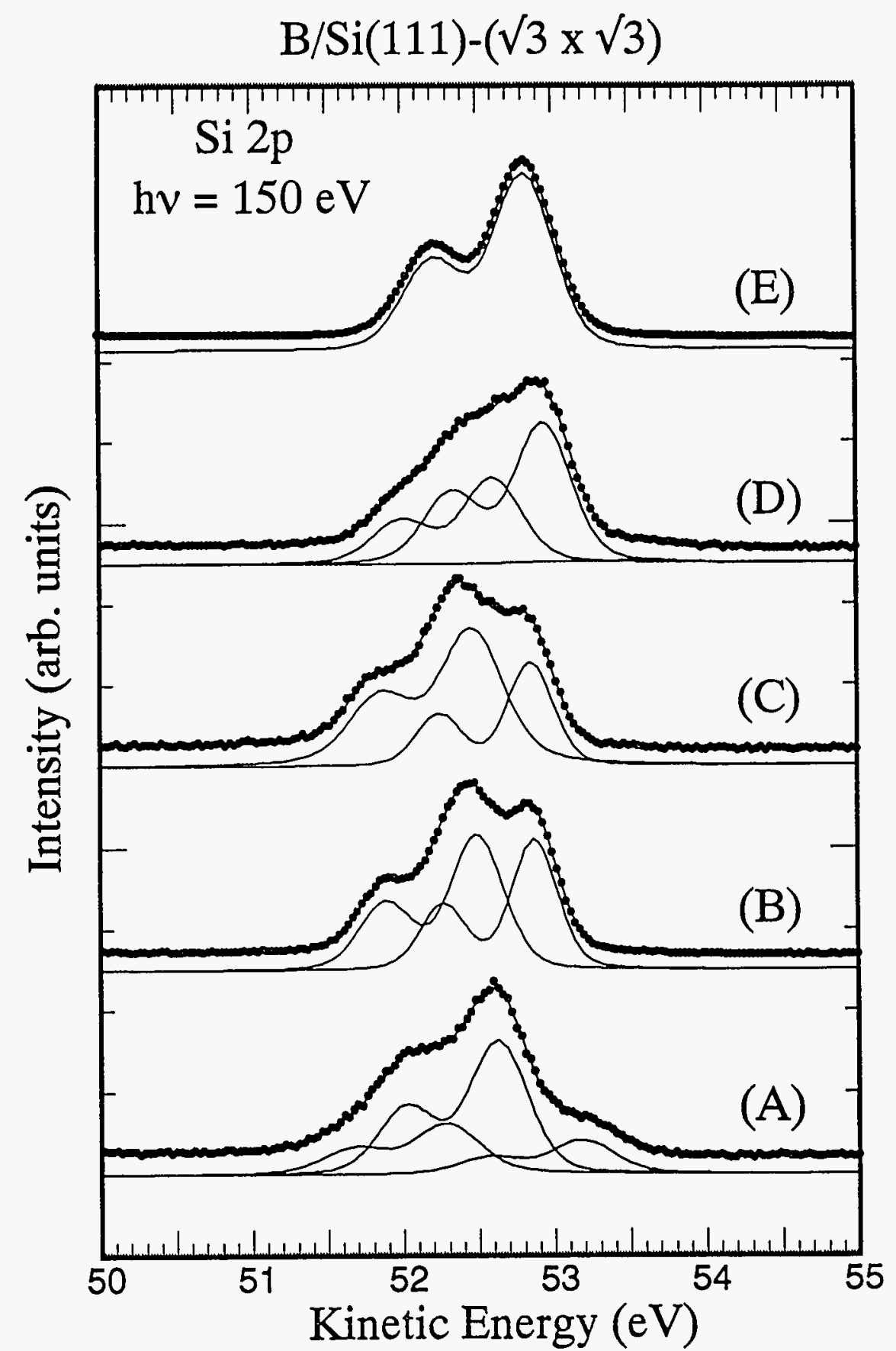

Fig. 2 


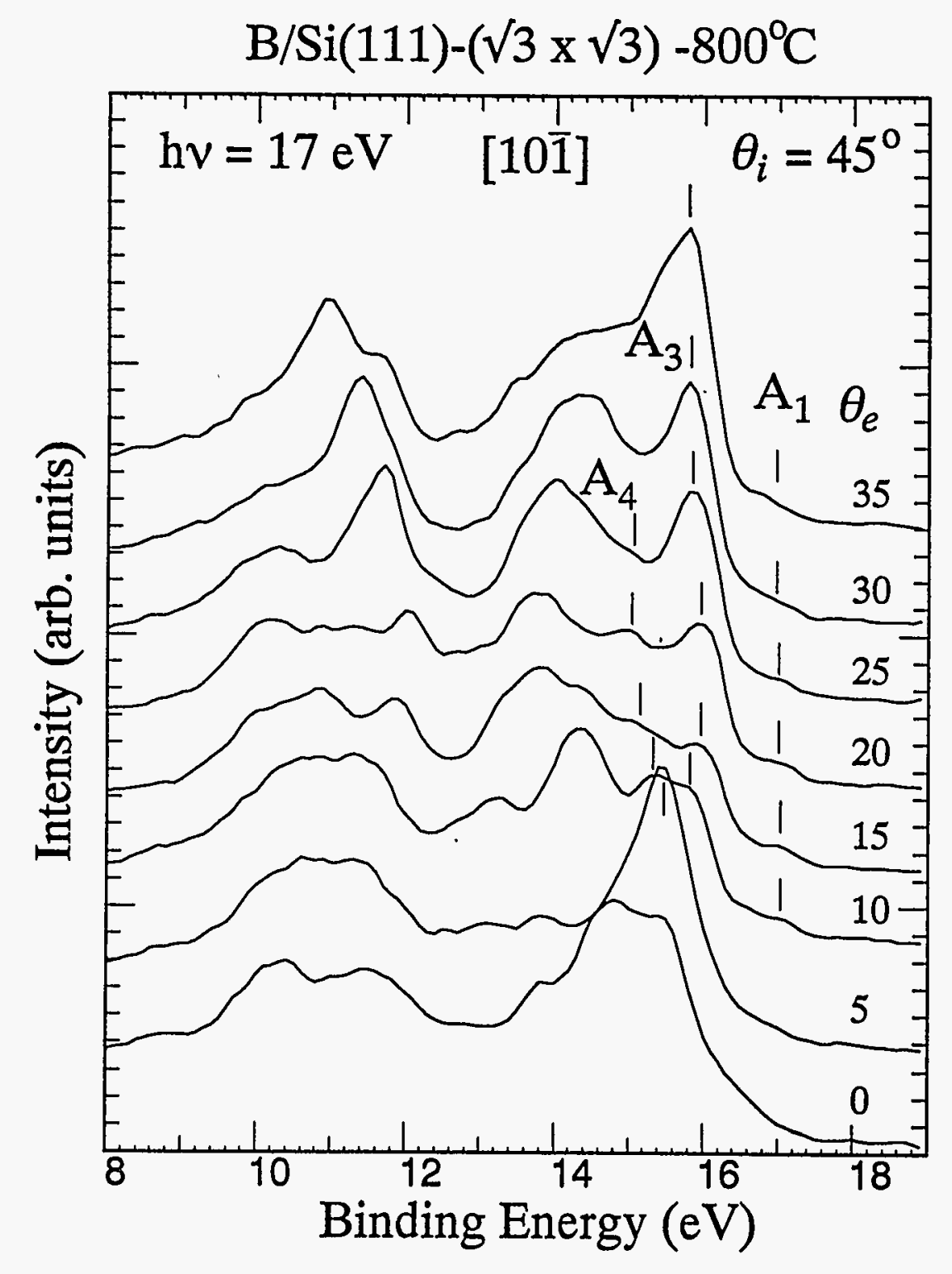

Fig. 3

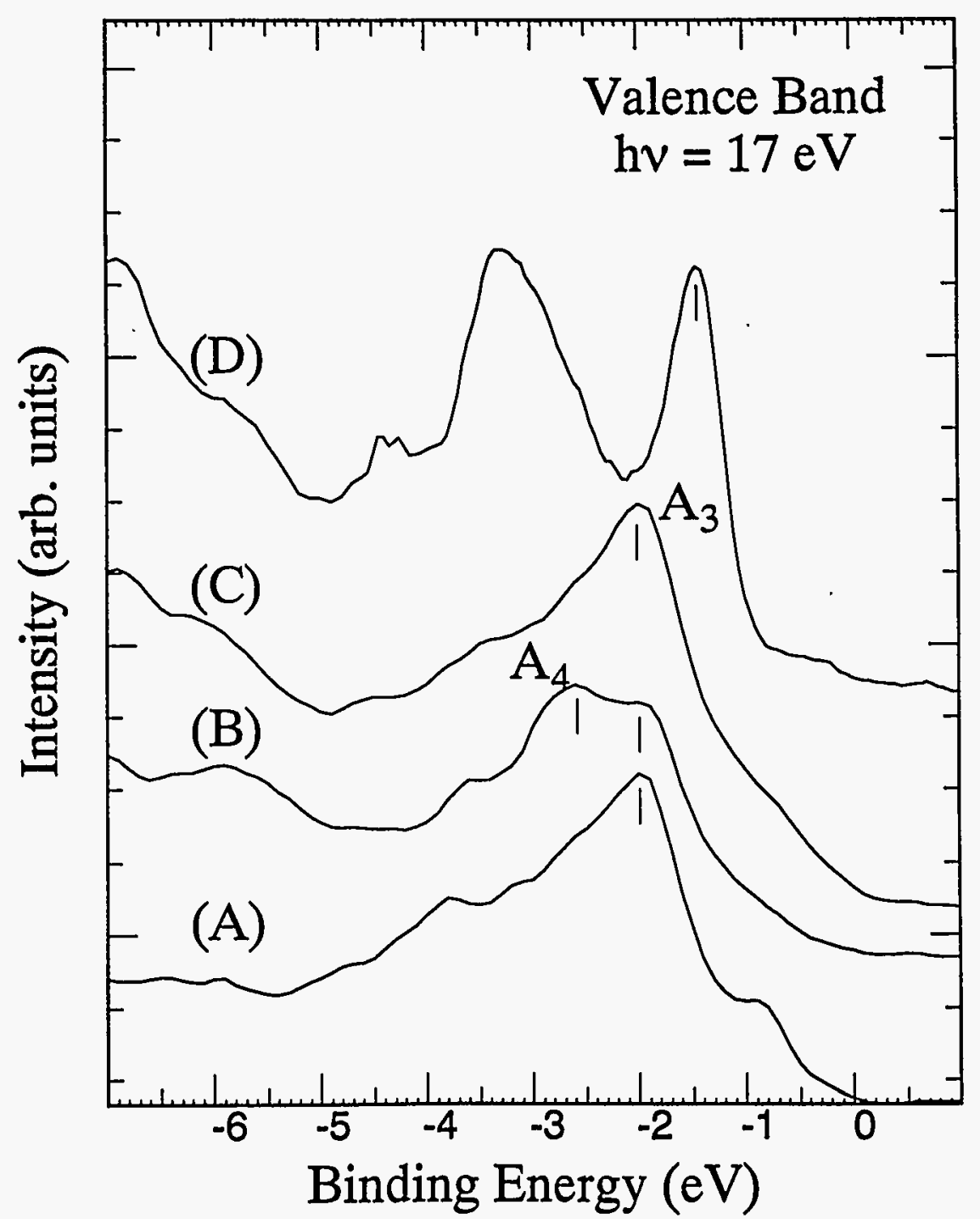

Fig. 4 